\title{
Analyzing and sense making of human factors in the Malaysian radiation and nuclear emergency planning framework
}

\begin{abstract}
The evolution of current Radiation and Nuclear Emergency Planning Framework (RANEPF) simulator emphasizes on the human factors to be analyzed and interpreted according to the stakeholderôs tacit and explicit knowledge. These human factor criteria are analyzed and interpreted according to the ñsense making theoryò and Disaster Emergency Response Management Information System (DERMIS) design premises. These criteria are corroborated by the statistical criteria. In recent findings, there were no differences of distributions among the stakeholders according to gender and organizational expertise. These criteria are incrementally accepted and agreed the research elements indicated in the respective emergency planning frameworks and simulator (i.e. 78.18 to 84.32 , p-value $<0.05$ ). This paper suggested these human factors criteria in the associated analyses and theoretical perspectives to be further acomodated in the future simulator development. This development is in conjunction with the proposed hypothesis building of the process factors and responses diagram. We proposed that future work which implies the additional functionality of the simulator, as strategized, condensed and concise, comprehensive public disaster preparedness and intervention guidelines, to be a useful and efficient computer simulation.
\end{abstract}

Keyword: Human factors; Radiation and nuclear emergency planning framework 